\title{
PRIMARY ACUTE ANGLE-CLOSURE GLAUCOMA DAMAGE TO CORNEA AND LENS*
}

\author{
BY \\ RONALD F. LOWE \\ From the Glaucoma Unit, the Royal Victorian Eye and Ear Hospital, Melbourne, \\ and the Ophthalmic Research Institute of Australia
}

ACUTE angle-closure glaucoma is a destructive disease unless it is controlled very quickly. The severe iris atrophy that may occur has been described by Winstanley (1961). The present paper illustrates the possibility of equally severe damage to the cornea and lens.

\section{Corneal Changes}

The following corneal conditions will be considered: (1) pressure oedema; (2) oedema following endothelial damage; (3) persistent folds in Descemet's membrane; (4) vascularization; (5) lipoid infiltration; (6) band-shape degeneration.

Oedema of the cornea caused by a sudden elevation of intra-ocular pressure is a diagnostic feature of acute angle-closure glaucoma. If the intra-ocular pressure rises to $40 \mathrm{~mm}$. $\mathrm{Hg}$ within some hours, corneal oedema is likely to occur, but corneal oedema may not accompany intra-ocular pressures of $80 \mathrm{~mm}$. $\mathrm{Hg}$ if the increase has occurred slowly over some months or years.

The acute pressure oedema tends to involve all the cornea, but not infrequently only the central area may be affected leaving the periphery clear. The pressure oedema affects especially the epithelium and superficial stroma so that it can be temporarily cleared with topical glycerine to permit gonioscopic and other examinations (Cogan, 1943). With reduction in intra-ocular pressure it disappears quickly.

When a severe attack of angle-closure glaucoma has persisted for twenty-four hours or more, the intra-ocular pressure may still be reduced rapidly by appropriate treatment, but the cornea remains oedematous. This type of oedema then shows as a thickening of the cornea with folds in Descemet's membrane. It is slow to clear, taking several days or even some weeks. It is less readily cleared with glycerine, and its persistence in the presence of low intra-ocular pressures is a striking feature. It is not cleared by hypertonic infusions such as 20 per cent. mannitol. It is probably caused by aqueous seeping through damaged endothelium and its clearing is dependent upon the recovery of these cells. Its regression is frequently assisted by topical corticosteroid eyedrops.

Folds in Descemet's membrane may persist long enough for them to develop hyaline appearances and they may then become permanent.

\footnotetext{
*Received for publication October 27, 1964.
} 
It is notable that despite the corneal oedema and the relatively frequent presence of cells in the aqueous, keratic precipitates are of only insignificant size and number, although fine pigment deposition is common.

Infrequently, resolution of an attack of acute glaucoma may occur spontaneously, or the patient may procrastinate long enough for the secretion of the ciliary body to diminish sufficiently for the intra-ocular pressure to fall to the level of comfort. Despite a permanently closed angle, the eye may whiten and the intra-ocular pressure stabilize around 40 or $50 \mathrm{~mm}$. Hg. The eye becomes blind from optic atrophy and the corneal oedema persists. From the conjunctiva, superficial vessels creep across the limbus (Fig. 1). Gradually they proliferate and thicken while deep vessels accompany fibrosis (Fig. 2).

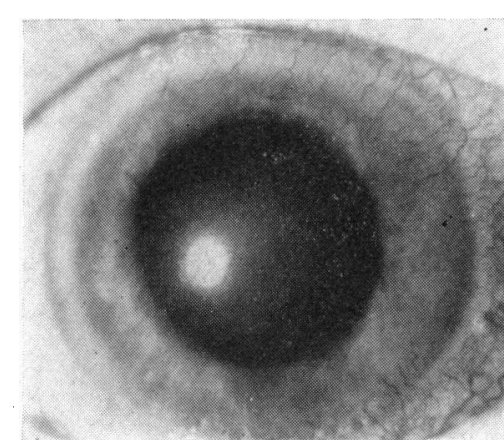

FIG. 1.-Chronic corneal oedema with fine vessels across the limbus; followed untreated angle-closure glaucoma.

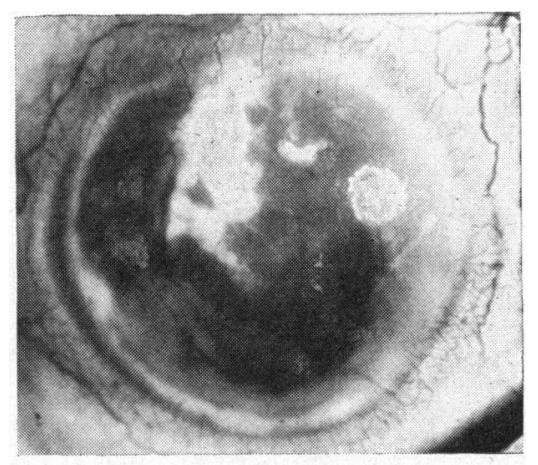

FIG. 3.-Heavy deposits of lipoid in cornea; acute angle-closure glaucoma unrelieved for one month.

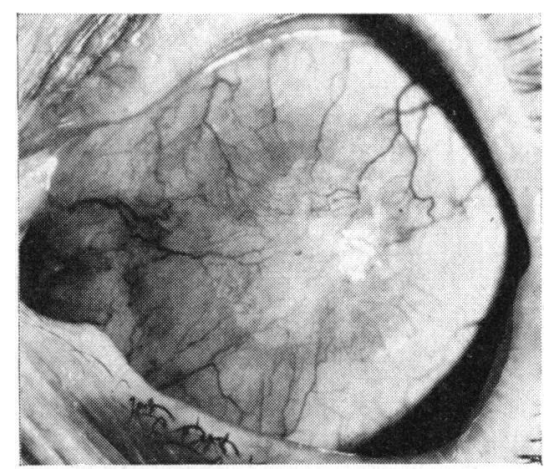

FIG. 2.-Corneal fibrosis and vascularization; followed untreated angle-closure glaucoma.

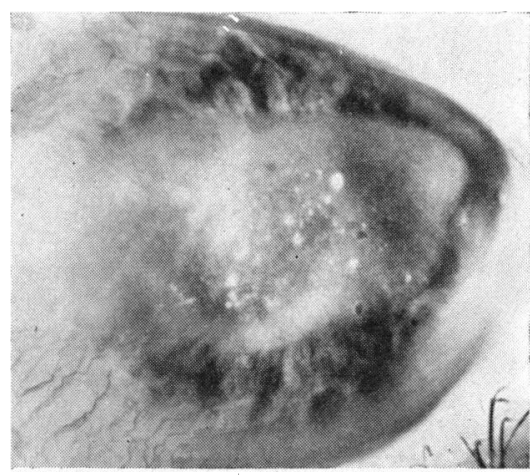

FIG. 4.-Band-shaped corneal degeneration; acute angle-closure glaucoma untreated.

In other eyes, as the corneal oedema diminishes, lipoid infiltration similar to an extensive arcus senilis, or as dense, irregular collections, may develop (Fig. 3). Occasionally, the oedema may clear completely, but the blind eye continues to deteriorate, and a large band-shaped corneal degeneration is found with its typical calcified spots (Fig. 4). 


\section{Lens Damage}

The following types of lens damage will be discussed: (1) glaukomflecken; (2) anterior capsular cataract; (3) pigment deposition and posterior synechiae; (4) cortical cataracts; (5) nuclear sclerosis; (6) damage from surgery.

Disseminated subcapsular cataracts of acute glaucoma (Figs 5 and 6) form a wellrecognized clinical entity, yet they have excited only a small bibliography since Vogt described them in 1930, and so beautifully illustrated them in his slit-lamp atlas in 1931. Jones (1959) described their changing appearances from their presentation as a continuous sheet in the subcapsular pupillary part of the lens, for seven to twenty-one days when they show as the dense white flecks described by Vogt. Many disappear before those that remain have clear lens fibres laid over the front of them and so appear to sink gradually into the lens cortex. Sparse representatives may be found by careful slit-lamp examination many years after the acute glaucoma attack.

Sugar (1957) thought that they did not occur beyond the pupil area, but in some patients who have had a full iridectomy or iridencleisis some flecks or patterns may be seen much further towards the lens equator, showing that they can develop beneath the iris. They have little effect upon vision.

Their presence is practically diagnostic of a past severe attack of acute glaucoma, but, rarely, they can occur after physical or chemical trauma (Pillat, 1957; Sugar, 1957), or they may develop in hypotensive eyes when anterior chambers collapse after surgery and the lens remains in contact with the cornea for three or four days (Lowe, 1964).

Glaukomflecken appear to be the result of coagulation of the tips of anterior lens fibres, and an unexplained phenomenon is the absence of their development in the fibres of the posterior lens cortex. There is no discernable reason why they develop in some lenses and not in others. Generally, they accompany the more severe attacks of acute glaucoma (very high sustained pressures), but although they may be seen within several hours of the onset of some severe attacks, when other apparently equally severe attacks are relieved only after some days no glaukomflecken
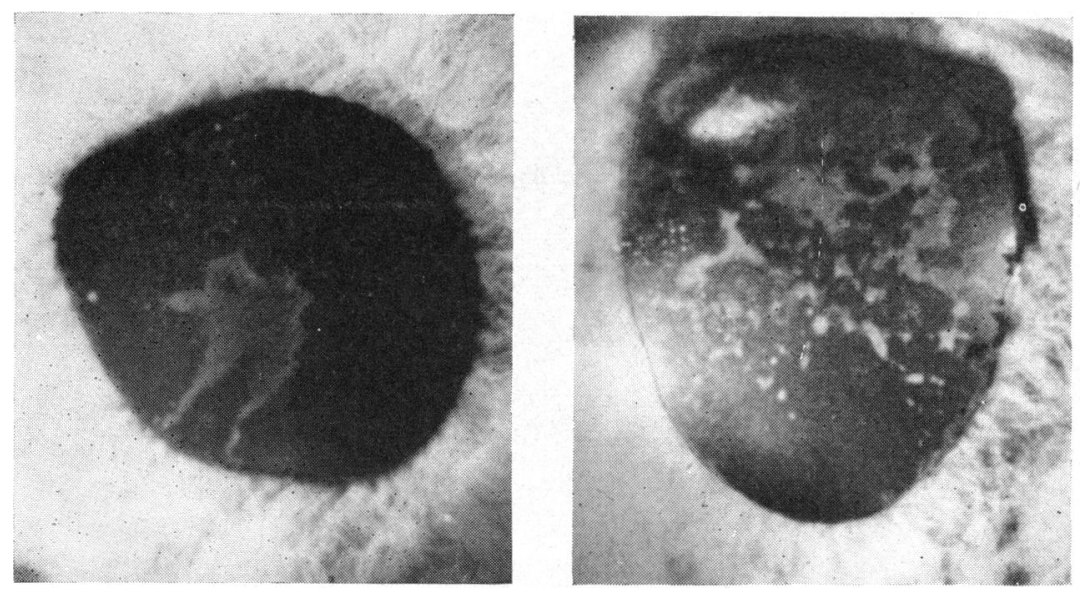

Fig. 5.-Examples of glaukomflecken with acute angle-closure glaucoma. 
are found. A similar variability in expected effects from time intensity occurs with the formation of peripheral anterior synechiae, other lens injuries, or iris atrophy.

The number and size of the glaukomflecken, and the area of their spread, vary considerably from case to case (Fig. 5), but the initial damage is always more extensive than that ultimately seen. Considerable recovery therefore occurs, whereas other forms of lens damage produced under similar circumstances tend to progress. The ability of the lens fibres to recover appears to diminish towards their extremities, thus producing the final sutural distribution of the flecks.

Occasionally, small sparse white spots resembling glaukomflecken may persist in the anterior surface of the lens and show no sinking by being covered with newformed anterior lens fibres (see Fig. 8). A collection of irregular rough opacities sometimes forms at the centre of the anterior lens surface (Fig. 6) to resemble pyramidal cataracts, which Treacher Collins (1892) showed "to consist in a localized proliferation and breaking up of the epithelial cells which line the hyaline capsule at the anterior pole".

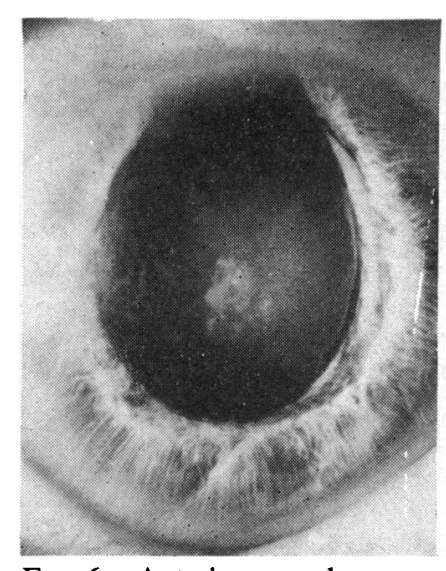

FIG. 6.-Anterior capsular cataract fromacute angle-closure glaucoma.

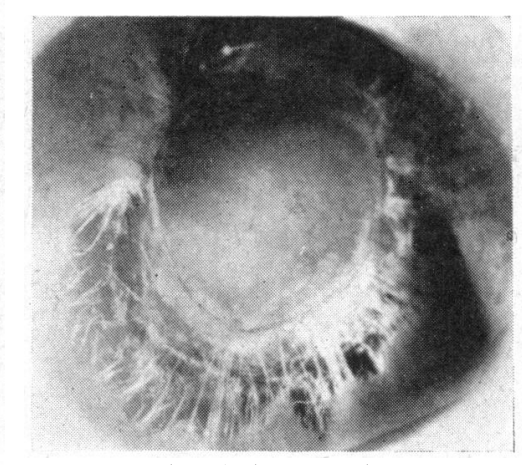

FIG. 7.-Fibrosis between iris and lens following acute angle-closure glaucoma.

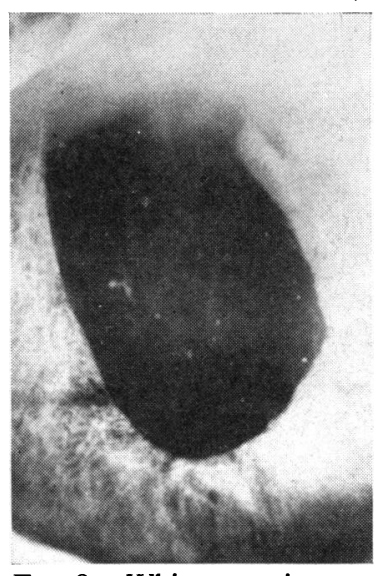

FIG. 8.-White spots in anterior lens capsule and streaky cortical lens opacities following acute angle-closure glaucoma.

Following acute glaucomatous iritis which leads to extensive posterior synechiae and iris atrophy, fibrosis may extend from the iris on to the anterior lens surface (Fig. 7). If lens extraction becomes necessary later, the breaking of this fibrosis to separate the lens from the iris almost invariably leads to a rupture of the anterior lens capsule, so that if total lens extraction is attempted this has to be performed piecemeal.

Cortical lens opacities are commonly seen after severe attacks of acute primary angle-closure glaucoma. They may present as faint irregular streaks within the anterior lens cortex (Fig. 8) or as dense irregular patterns on a milky background (Fig. 9). These opacities almost invariably progress; the most severe cause a quick loss of vision, whereas lenses showing relatively minor initial damage frequently deteriorate within two to four years. Careful lens extraction is usually possible without undue complications. 


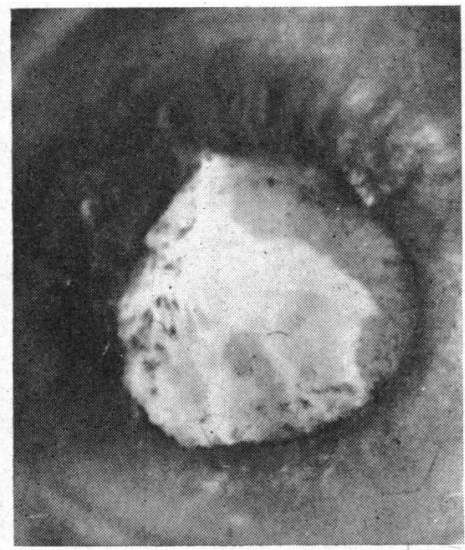

FIG. 9.-Patterns in anterior lens cortex and mature cataract following acute angle-closure glaucoma.

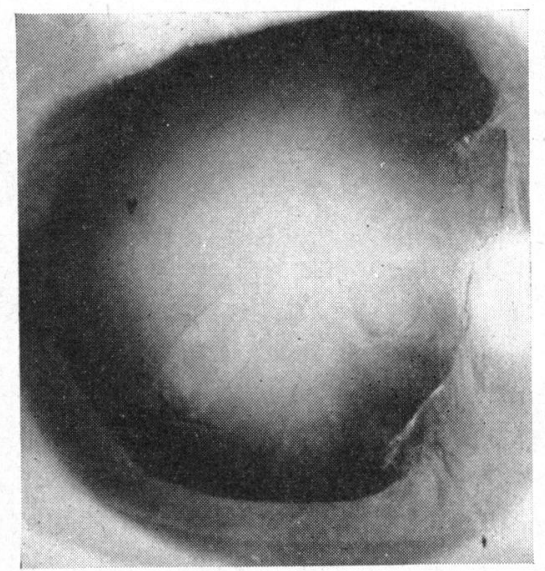

Fig. 10.-Heavy sclerosis of lens nucleus following acute angle-closure glaucoma.

Other lenses develop a heavy nuclear sclerosis accompanied by a dense central clouding (Fig. 10). Increased myopia and gradual reduction of vision are to be expected.

Lens damage may be caused by surgery performed to relieve or prevent relapse of the acute glaucoma. Incisions with a von Graefe knife have the advantage of permitting slow decompression and giving a large internal opening of the incision, but the equator of the lens is liable to be injured. Small linear opacities (Fig. 11) tend to become stationary and have little effect on vision but larger lens injuries can be disastrous. Rapid release of high pressures is likely to lead to lens dislocation, especially upwards and against the operation site.

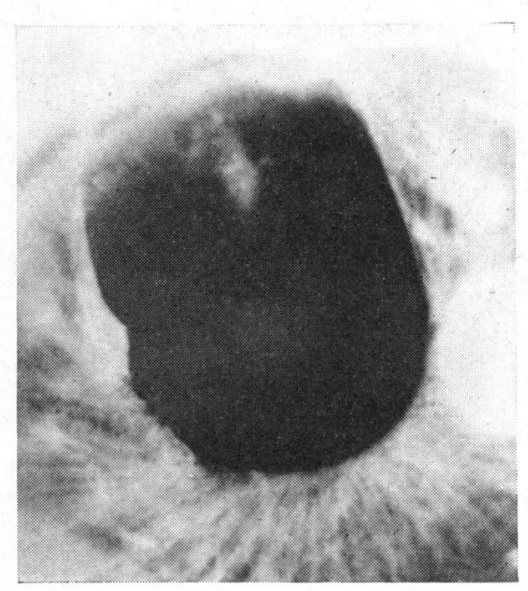

FIG. 11.-Peripheral lens opacities caused by Graefe knife injury during iridectomy for acute angle-closure glaucoma.

\section{Discussion}

Enough technical knowledge has been gathered for acute angle-closure glaucoma to have an almost uniformly favourable outcome, providing patients present early enough, general practitioners recognize it, and ophthalmologists implement modern ideas in its management.

Most attacks of acute angle-closure glaucoma strike without previous warning, so that only education of the public in recognizing the signs can reduce the grave procrastination that causes so much damage before medical advice is sought. General practitioners need more enlightenment, so that severely red eyes are not given a trial treatment with topical antibiotics and steroids before the presence of 
serious eye disease is realized. Ophthalmologists should apply intense therapy to control the attacks rapidly, aiming to halt the destructive processes.

Many ophthalmologists are still not convinced of the necessity for prophylactic surgery, either upon the companion eye with its 75 per cent. risk of an acute angleclosure attack, or for the recurrent attacks of subacute angle-closure glaucoma before the destructive acute attack occurs. Ophthalmologists who are apprehensive of performing prophylactic operations should send their patients to surgeons whose skill can give appropriate eyes the safety of peripheral iridectomies.

Despite the advances that have been made in the concepts, diagnosis, and treatment of acute angle-closure glaucoma, the disease still leaves a trail of destruction in its wake.

\section{Summary}

Severe damage to the cornea or lens may occur with acute angle-closure glaucoma that is not quickly relieved. Examples are illustrated. A practically uniformly favourable outcome could be achieved in angle-closure glaucoma but for procrastination, wrong diagnosis, or inadequate therapy.

The photographs were taken by the clinical photographers of the Royal Victorian Eye and Ear HospitalMr. T. Cottier and Mrs. G. van den Brenk. The patients were collected in the Glaucoma Unit of the hospital during Research Project No. 13 of the Royal Victorian Eye and Ear Hospital and Research Project No. 14 of the Ophthalmic Research Institute of Australia. I wish to thank my colleagues for permission to examine their patients and for access to their records.

\section{REFERENCES}

Cogan, D. G. (1943). Amer. J. Ophthal., 26, 551.

Collins, E. T. (1892). Trans. ophthal. Soc. U.K., $12,89$.

JONES, B. R. (1959). Ibid., 79, 753.

Lowe, R. F. (1964). Orient. Arch. Ophthal., 2, 267.

Pillat, A. (1957). Forsch. und Praxis, 10, 13 (quoted by Jones, B. R., 1959).

SugAR, H. S. (1957). "The Glaucomas", 2nd ed. Hoeber, New York.

VoGt, A. (1930). Klin. Mbl. Augenheilk., 85, 586. (1931). "Lehrbuch und Atlas der Spaltlampenmikroskopie des Lebenden Auges", 2nd ed., vol. 2. Springer, Berlin.

Winstanley, J. (1961). Trans. ophthal. Soc. U.K., 81, 23. 